\title{
Wnts Influence the Timing and Efficiency of Oligodendrocyte Precursor Cell Generation in the Telencephalon
}

\author{
Abraham J. Langseth, ${ }^{1,3}$ Roeben N. Munji, ${ }^{4 \star}$ Youngshik Choe, ${ }^{4 \star}$ Trung Huynh, ${ }^{4}$ Christine D. Pozniak, ${ }^{4}$ \\ and Samuel J. Pleasure ${ }^{1,2,3,4}$ \\ Programs in ${ }^{1}$ Neuroscience and ${ }^{2}$ Developmental Biology, ${ }^{3}$ Eli and Edythe Broad Center of Regeneration Medicine and Stem Cell Research, and ${ }^{4}$ Department \\ of Neurology, University of California, San Francisco, San Francisco, California 94158
}

Oligodendrocyte precursor cells (OPCs) are generated from multiple progenitor domains in the telencephalon in developmental succession from ventral to dorsal. Previous studies showed that Wnt signaling inhibits the differentiation of OPCs into mature oligodendrocytes. Here we explored the hypothesis that Wnt signaling limits the generation of OPCs from neural progenitors during forebrain development. We manipulated Wnt signaling in mouse neural progenitor cultures and found that Wnt signaling influences progenitors cell autonomously to alter the production of OPCs, and that endogenous Wnt signaling in these cultures limits the efficiency of generating OPCs from neural progenitors. To examine these events in vivo, we electroporated a soluble Wnt inhibitor or a dominant-negative transcriptional regulator into embryonic mouse neocortical ventricular zone before the usual onset of OPC production and showed that decreasing Wnt signaling in cortical progenitors results in early production of OPCs. Our studies indicate that Wnt signaling influences the timing and extent of OPC production in the developing telencephalon.

\section{Introduction}

Oligodendrocyte precursor cells (OPCs) are generated from progenitor zones in the forebrain beginning at various times during embryonic development (Kessaris et al., 2006). The mechanisms regulating the spatial and temporal production of OPCs have not been clearly elucidated; in particular, it is not clear why OPCs appear dorsally at later stages than ventrally. Sonic Hedgehog regulates the production of OPCs from ventral progenitor zones, but the signaling pathways that regulate the dorsal generation of OPCs are only beginning to be elucidated (Richardson et al., 2006).

Wnts are secreted glycoprotein ligands that regulate development of dorsal CNS structures (Lee and Jessell, 1999). Wnts have prominent roles in progenitor cell proliferation, cell lineage, and differentiation decisions (Freese et al., 2010). In the telencephalon, Wnt signaling plays a prominent dorsalizing role (Lee et al., 2000; Kim et al., 2001; Backman et al., 2005; Zhou et al., 2006; Machon et al., 2007; Chenn, 2008; Gulacsi and Anderson, 2008; Freese et al., 2010). Some have suggested that the dorsal-ventral gradient of Wnt signaling in the cortex is related to the gradient of cortical neuronal differentiation (Machon et al., 2007). Studies have shown that Wnts inhibit the differentiation of OPCs into

Received April 15, 2010; revised Aug. 4, 2010; accepted Aug. 15, 2010.

This work was supported by the California Institute for Regenerative Medicine, National Multiple Sclerosis Society, and the family of Glenn W. Johnson, Jr. (S.J.P.) and a National Science Foundation Graduate Research Fellowship (A.J.L.). We thank Guangnan Li and Gerrit J. P. Dijkgraaf for constructs.

${ }^{*}$ R.N.M. and Y.C. contributed equally to this work.

Correspondence should be addressed to Samuel J. Pleasure, Department of Neurology, University of California, San Francisco, Mission Bay, Box 2822, 1550 4th Street, Room 448C, Rock Hall, San Francisco, CA 94158. E-mail: sam.pleasure@ucsf.edu.

C. D. Pozniak's present address: Genentech, Inc., 1 DNA Way, South San Francisco, CA 94080.

DOI:10.1523/JNEUROSCI.1934-10.2010

Copyright $\odot 2010$ the authors $\quad$ 0270-6474/10/3013367-06\$15.00/0 oligodendrocytes and thereby regulate myelination and remyelination following injury (Shimizu et al., 2005; Fancy et al., 2009; Ye et al., 2009).

These factors raise the possibility that Wnt ligands in the dorsal forebrain influence the generation of OPCs from neural progenitors, thereby controlling the timing of OPC production. A recent report (Ye et al., 2009) demonstrated that disrupting $\beta$-catenin changes the number of OPCs in the developing CNS and that Wnts work with histone deacetylases to regulate oligodendrocyte differentiation. However, this study leaves a number of unanswered questions since it relied on the Olig1-cre driver line expressed very early in the forebrain and on manipulating $\beta$-catenin directly. Reliance on loss-of-function alleles of $\beta$-catenin does not allow exclusion of non-Wnt functions of $\beta$-catenin in the ventricular zone such as cell adhesion. Also, reliance on dominantly active $\beta$-catenin can lead to effects that are not normally regulated by Wnt signaling in vivo.

We wanted to determine whether Wnt signaling influences the generation of OPCs from neural progenitor cells and whether it plays a role in the delayed appearance of OPCs from dorsal telencephalic regions. We regulated the levels of extracellular Wnt ligand and cell-autonomously altered Wnt signaling. We show that Wnt signaling negatively regulates the specification of OPCs from neural progenitors and that inhibition of Wnt signaling drives a robust prescheduled increase in the production of OPCs in the cortex. This indicates that Wnts influence the timing and balance of OPC production from ventricular zone progenitors.

\section{Materials and Methods \\ Animals \\ All animal protocols were approved by the University of California, San Francisco Institutional Animal Care and Use Committee. P5 CD1s}




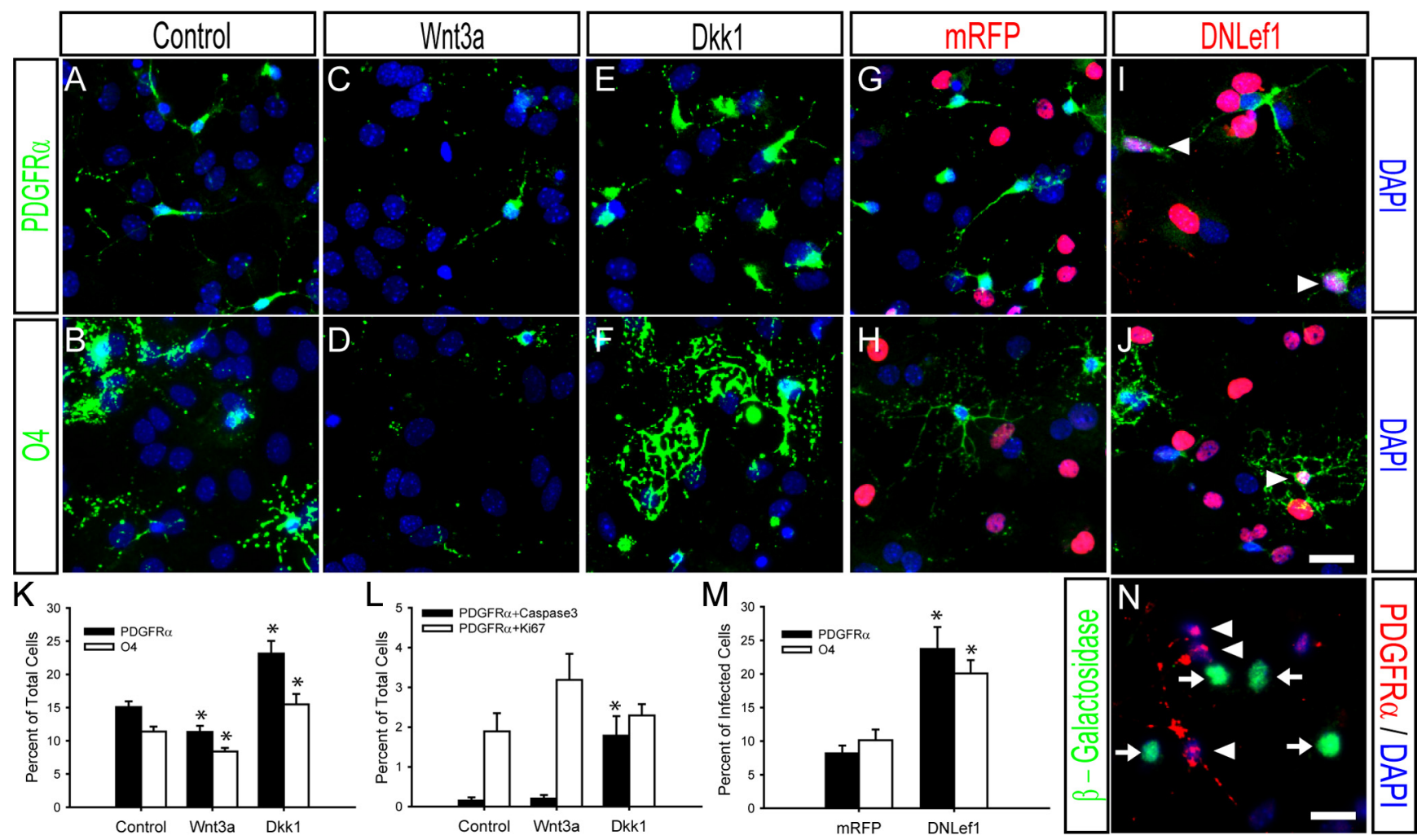

Figure 1. High Wnt tone decreases OPC production from neural progenitors, while low Wnt tone enhances production. Application of Wnt3a to neural progenitor cultures for $4 \mathrm{~d}$ decreased the number of PDGFR $\alpha$ - $(\boldsymbol{C}, 11.34 \pm 0.93 \%$, mean percentage of total cells \pm SE) and $04-(\boldsymbol{D}, 8.40 \pm 0.53 \%)$ positive cells and application of Dkk1 antagonist increased the number of OPCs $(\boldsymbol{E}, 23.14 \pm 1.88 \% ; \boldsymbol{F}, 15.48 \pm 1.56 \%)$ compared to control cultures $(\boldsymbol{A}, 15.06 \pm 0.89 \% ; \boldsymbol{B}, 11.38 \pm 0.75 \%)$. This is graphed in $\boldsymbol{K}$. This is not a result of proliferation (Ki-67) or apoptosis (activated Caspase3) of OPCs, as the percentage of proliferating OPCs was greatest in the Wnt3a group (3.19 $\pm 0.65 \%$, control $=1.89 \pm 0.46 \%$, Dkk1 $=2.30 \pm$ $0.27 \%$ ) and the percentage of apoptotic OPCs was greatest in the Dkk1 group and significantly greater than control (Dkk1 $=1.78 \pm 0.49 \%$, control $=0.15 \pm 0.08 \%$ Wnt $3 \mathrm{a}=0.20 \pm$ $0.09 \%)(\boldsymbol{L})$. Retroviral infection of neural progenitors with DNLef1-mRFP results in a significant increase in PDGFR $\alpha$-positive cells $(\boldsymbol{I}, 23.74 \pm 3.26 \%)$ and 04 -positive cells $(\boldsymbol{J}, 20.07 \pm$ $1.99 \%)$ by $4 \mathrm{~d}$ after infection compared to mRFP control infection $(\mathbf{G}, 8.18 \pm 1.18 \% ; \boldsymbol{H}, 10.12 \pm 1.62 \%)$. Arrowheads indicate infected OPCs. This is graphed in $\boldsymbol{M}$. $N=3$ for all groups. Neural progenitor cells were cultured from BAT-gal mice. $\boldsymbol{N}, 6.32 \pm 1.13 \%$ of PDGFR $\alpha$-positive BAT-gal cells (arrowheads) show detectable Wnt signaling (arrows). Scale bars, $10 \mu \mathrm{m}$. ${ }^{*} p \leq 0.05$ for ANOVA in $\boldsymbol{K}$ and Student's $t$ test in $\boldsymbol{L}$ and $\boldsymbol{M}$.

(Charles River) were used for cultures. BAT-gal mice (Maretto et al., $2003)$ were used for $\beta$-galactosidase ( $\beta$-gal) expression analysis and for DNLef1 electroporations. C57BL/6 mice (The Jackson Laboratory) were used for Dkk1 electroporations. Males and females were used for all experiments.

\section{Retroviral production}

pLNCX2 (Clontech) was modified to include a new multiple cloning site and histone $\mathrm{H} 2 \mathrm{~B}$ fused to monomeric red fluorescent protein (pLH2BmRFP). A dominant-negative Lef1 (DNLef1) construct [Lef1 (Clontech) with a truncated $\mathrm{N}$ terminus] was inserted. Retrovirus was produced in GP2-293 cells (Clontech) and concentrated using standard methods.

\section{In utero electroporations}

See Li et al. (2008) for method. Electroporations (EPs) were targeted to the lateral cortex at E13.5 or E15.5 and collected 4 or $6 \mathrm{~d}$ later. The DNLef1 construct was inserted into pCAGGS (chicken $\beta$-actin cytomegalovirus promoter-driven expression). pCIG (GFP) [from A. McMahon, Harvard University, Cambridge, MA (Megason and McMahon, 2002)] was used as a control. pCIG-Dickkopf-1 was provided by L. W. Burrus, San Francisco State University, San Francisco, CA.

\section{Immunohistochemistry and X-gal staining}

Brains were processed using standard methods. Cells and tissue sections were stained using standard protocols with the following: rat anti-PDGFR $\alpha$ (BD Biosciences PharMingen,1:500 for tissue and 1:800 for cells), rabbit anti-Olig2 (Millipore, 1:1000 for tissue), mouse anti- $\beta$-gal (Promega, 1:2000 for tissue and 1:6000 for cells), and mouse anti-O4 (Sigma, 1:30 for cells). Fluorescent secondary antibodies (1:1000, Invitrogen) and DAPI (1:3000, Sigma) were used. For X-gal staining, floating sections were stained with X-gal substrate (Invitrogen).

\section{Cell culture}

Neural progenitor cells (NPCs) were from lateral ventricle SVZ tissue of P5 CD1s. Tissue was dissociated with 0.1\% Trypsin (Worthington) and 0.1\% DNase1 (Roche). DMEM/F12 (50:50, Invitrogen) was supplemented with $4.0 \mu \mathrm{l}$ of $3 \mathrm{~mm}$ selenium, $4.0 \mu \mathrm{l}$ of $2 \mathrm{~mm}$ progesterone, $10 \mu \mathrm{l}$ of $2 \mu \mathrm{g} / \mathrm{ml}$ heparin (all from Sigma), $10.8 \mathrm{ml}$ of $30 \%$ glucose, $8.1 \mathrm{ml}$ of $7.5 \% \mathrm{NaHCO}_{3}, 2.5 \mathrm{ml}$ of $1 \mathrm{~m}$ HEPES, $5 \mathrm{ml}$ of $200 \mathrm{~mm}$ glutamine, $5 \mathrm{ml}$ of penicillin-streptomycin, and $2 \mathrm{ml}$ of Fungizone for a total volume of 50 ml. Media containing EGF (Sigma, $10 \mathrm{ng} / \mathrm{ml}$ ), FGF (Sigma, $20 \mathrm{ng} / \mathrm{ml}$ ), and B27 (Invitrogen) was added. Cells were plated at 80,000 cells per 25 $\mathrm{cm}^{2}$ flask (Corning), grown in $5 \mathrm{ml}$ of complete media plus growth factors and B27. Passage 1 or 2 NPCs were used.

Wnt treatments. NPCs were dissociated with $0.05 \%$ trypsin-EDTA, washed with PBS, pelleted, and resuspended in $500 \mu \mathrm{l}$ of complete media with $2 \%$ FBS. The cells were plated on laminin-coated $(1 \mathrm{mg} /$ ml, Invitrogen) chamber slides (Nunc) at 37,500 cells/well. Mouse Wnt3a $(0.15 \mathrm{ng} / \mu \mathrm{l})$, Dickkopf1 (Dkk1, $0.03 \mathrm{ng} / \mu \mathrm{l})$, or an equivalent volume of PBS was added to each well. One half of the media was replaced each day. The cultures were analyzed after $4 \mathrm{~d}$ in vitro (DIV).

Retroviral infection. NPCs were dissociated, washed with PBS, pelleted, and resuspended in $500 \mu \mathrm{l}$ of complete media containing EGF, FGF, B27, and $5 \mu \mathrm{g} / \mathrm{ml}$ polybrene (Sigma). Concentrated virus $(50-100 \mu \mathrm{l} ; \sim 1 \times$ $10^{5}$ transducing units) was added to $\sim 300,000$ cells, which were spin 
infected for $90-120 \mathrm{~min}$ at $170 \times \mathrm{g}$. The cells were plated in complete media containing growth factors in a $25 \mathrm{~cm}^{2}$ flask for $24 \mathrm{~h}$ to allow integration and expression. Cells were resuspended in complete media with $2 \%$ FBS and plated on chamber slides at 37,500 cells/well and analyzed after 4 DIV.

\section{Image analysis and quantification}

Three to four biological replicates of each experiment were performed. Ten $20 \times$ magnification images were quantified for each replicate of each cell culture condition. Cell counts from 3-4 coronal sections of each EP brain were averaged.

\section{Statistical analysis}

Results are expressed as mean \pm SEM. Data were analyzed using twotailed Student's $t$ test with unequal variance. Multiple comparisons were made using ANOVA with a post hoc Holm-Sidak test. Any value of $p \leq$ 0.05 was considered significant.

\section{Results}

\section{Wnt signaling regulates OPC generation from telencephalic} progenitor cells

To test the role of Wnt signaling in the generation of OPCs, we cultured neural progenitors from the telencephalons of perinatal mice. We plated the progenitors and exposed them to FBS to induce differentiation along with soluble mouse Wnt3a ligand (Fig. 1C,D), soluble mouse Dickkopf1 (Dkk1), a Wnt inhibitor (Fig. 1E,F), or an equivalent volume of PBS as a control (Fig. $1 A, B)$ for 4 DIV and then labeled the cells with the OPC markers PDGFR $\alpha$ and O4. Treatment with Wnt3a caused a 25\% decrease in the percentage of OPCs, and inhibition of Wnt signaling by Dkk1 caused a 54\% increase in the percentage of OPCs compared to controls (Fig. $1 \mathrm{~K}$ ). This was not a result of changes in the proliferation or apoptosis of OPCs (Fig. $1 \mathrm{~L}$ ). These results indicate that Wnt signaling influences the generation of OPCs from progenitor cells.

To determine whether this is a cell-autonomous effect, we infected progenitors with a retrovirus encoding a dominantnegative form of Lef1 (DNLef1-mRFP) (Fig. 1I, J, arrowheads indicate infected OPCs) or a nuclear mRFP control vector (Fig. 1G,H). PDGFR $\alpha$ and O4 labeling revealed a doubling in the percentage of infected OPCs $4 \mathrm{~d}$ after infection (Fig. $1 M$ ). To ensure that this effect was actually autonomous, we counted the OPCs among uninfected cells and found no difference between control and experimental conditions (supplemental Fig. 1, available at www.jneurosci.org as supplemental material).

Our data indicate that Wnt signaling influences the production of OPCs from neural progenitors, but we wanted to determine whether high levels of Wnt signaling are inconsistent with the decision of neural progenitors to generate OPCs. To examine this, we cultured neural progenitors from a Wnt signaling reporter mouse (BAT-gal). These cultures were differentiated for 4 DIV and then labeled with antibodies for $\beta$-gal (Fig. $1 N$, arrows) and PDGFR $\alpha$ (arrowheads). Similar to previously published reports (Fancy et al., 2009; White et al., 2010), we found that $\beta$-gal was expressed in $\sim 6 \%$ of the OPCs in our cultures. This indicates that part of the normal differentiation program for OPCs involves a lack of significant Wnt signaling at the time of OPC specification.

\section{Wnt signaling regulates the timing of OPC generation during development}

A previous report (Kessaris et al., 2006) showed that there are multiple waves of OPC production beginning ventrally and that cortical production does not begin until $\sim \mathrm{P} 0$. We wanted

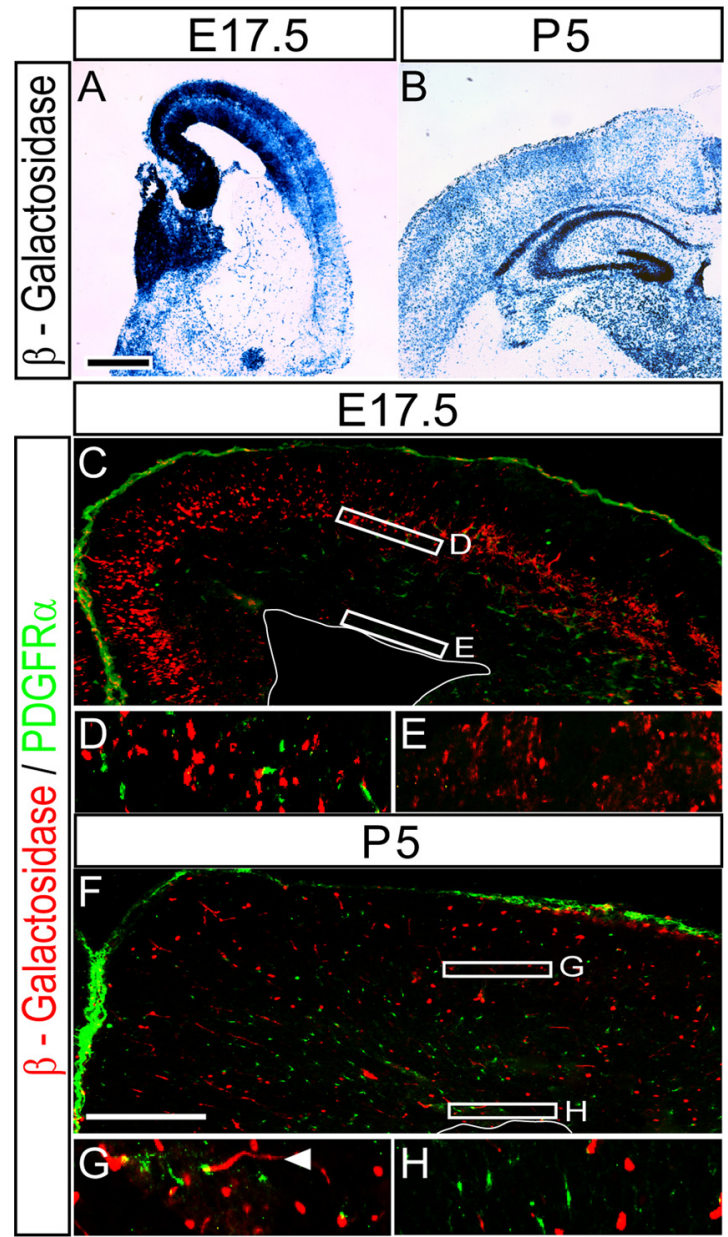

Figure 2. Wnt tone decreases in progenitor zones before they produce OPCS. $\boldsymbol{A}, \boldsymbol{B}, \mathrm{BAT}$-gal sections were treated with $\mathrm{Xgal}$. $\boldsymbol{A}$, Cortical cells have high Wnt tone during neurogenesis (E17.5). $\boldsymbol{B}$, Wnt tone in the cortex and SVZ is significantly decreased by the time of OPC production (P5). $\mathbf{C}-\boldsymbol{H}, \boldsymbol{\beta}$-Galactosidase and PDGFR $\alpha$ immunohistochemistry of BAT-gal forebrain. $\boldsymbol{C}$ At E17.5, cells near the ventricle and in the cortex show strong Wnt signaling. $\boldsymbol{D}, \boldsymbol{E}$, Highermagnification images. $\boldsymbol{F}$, By P5, only cortical blood vessels and scattered cortical cells show strong Wnt tone. $\boldsymbol{G}, \boldsymbol{H}$, Higher-magnification images. Most PDGFR $\alpha$-positive cells do not have strong Wnt signaling. Lines indicate lateral ventricle. Arrowhead indicates blood vessel. Scale bars, $500 \mu \mathrm{m}$.

to assess global levels of Wnt signaling in the cortical progenitor zone before and after the onset of cortical OPC production. X-gal staining of BAT-gal brains revealed that Wnt signaling is very high in cortical progenitors at E17.5 (before cortical OPC production) and decreased by P5 when OPC production is prominent (Fig. $2 A, B$ ). $\beta$-gal and PDGFR $\alpha$ labeling (Fig. 2C,F) at E17.5 showed many cells in the cortical $\mathrm{VZ/SVZ/IZ} \mathrm{and} \mathrm{many} \mathrm{presumptive} \mathrm{neurons} \mathrm{in} \mathrm{the} \mathrm{cortex} \mathrm{that}$ have strong Wnt signaling (Fig. 2D,E), but at P5 the number of cells near the ventricle with strong Wnt signaling was decreased and the OPCs in the cortex do not show evidence of strong Wnt signaling (Fig. $2 G, H$ ). At E17.5, many OPCs that were generated from ventral progenitor domains had migrated to the cortex and were within the region of strong Wnt signaling (Fig. 2C,D) (Kessaris et al., 2006), but no OPCs are generated from the cortical progenitor zone at this age (Fig. $2 E$ ) (Kessaris et al., 2006). We replicated the lineage tracing of cortically derived OPCs performed previously (Kessaris et al., 2006) using Emx1-Cre and found that few OPCs in the cortex at $\mathrm{P} 0$ are derived from the cortical progenitor domain but that 
by $\mathrm{P} 4$, cortically derived OPCs were abundant (data not shown). At P5 OPCs were throughout the cortex (Fig. $2 F$ ), including in the progenitor zone, which showed reduced Wnt signaling (Fig. $2 \mathrm{H})$. At this stage, many of the cells that showed the strongest Wnt signaling are associated with presumptive blood vessels (Fig. 2G, arrowhead) (Franco et al., 2009).

\section{Manipulation of Wnt signaling in progenitors changes the timing of OPC generation}

The timing of decreasing Wnt signaling in cortical progenitors raises the possibility that OPC generation from the cortex is limited by the strong Wnt signaling present prenatally and that this is a factor in determining the onset of OPC production. To test this idea we performed in utero EPs of a DNLef1 or control mRFP construct into neocortical progenitors at E15.5 and collected the embryos 4 d later at P0.5 (Fig. $3 A, B$, arrowheads indicate EP OPCs). This approach alters the level of Wnt signaling in primary multipotent cortical progenitors and addresses whether Wnt signaling influences production of OPCs from these cells. PDGFR $\alpha$ and Olig2 antibody labeling showed that there was a $66 \%$ increase in the percentage of EP OPCs after DNLef1 electroporation compared to controls (Fig. 3E). No changes in the mitotic fraction or apoptosis of EP OPCs were observed (data not shown). This indicates that shutting off the transcriptional output of the Wnt pathway in progenitors drives an increase in the production of OPCs.

To determine whether the endogenous levels of Wnt ligand present in the brain limit early OPC production, we performed EPs of Dkk1 or control GFP (Fig. $3 C, D$, white boxes indicate region of quantification) at E15.5 and collected the brains $6 \mathrm{~d}$ later. PDGFR $\alpha$ and Olig2 antibody labeling showed a $61 \%$ increase in the number of OPCs in the EP region (Fig. $3 F$ ). This manipulation is not cell autonomous and influences both the neural progenitors and the OPCs already present in the cortex. Since others have shown that Wnt signaling is observed in a subset of cortical OPCs and may drive their proliferation (Fancy et al., 2009; White et al., 2010), the Dkk1 EP may decrease proliferation of OPCs already present in the cortex. Therefore, the decrease in Wnt signaling in the progenitor domain must cause an increase in the number of OPCs produced, which overcomes their decreased drive to proliferate. This indicates that decreasing Wnt signaling in progenitors or decreasing their exposure to endogenous levels of Wnt ligand in the environment leads to an early onset of cortical OPC generation. $t$ test in $\boldsymbol{E}$ and $\boldsymbol{F}$.

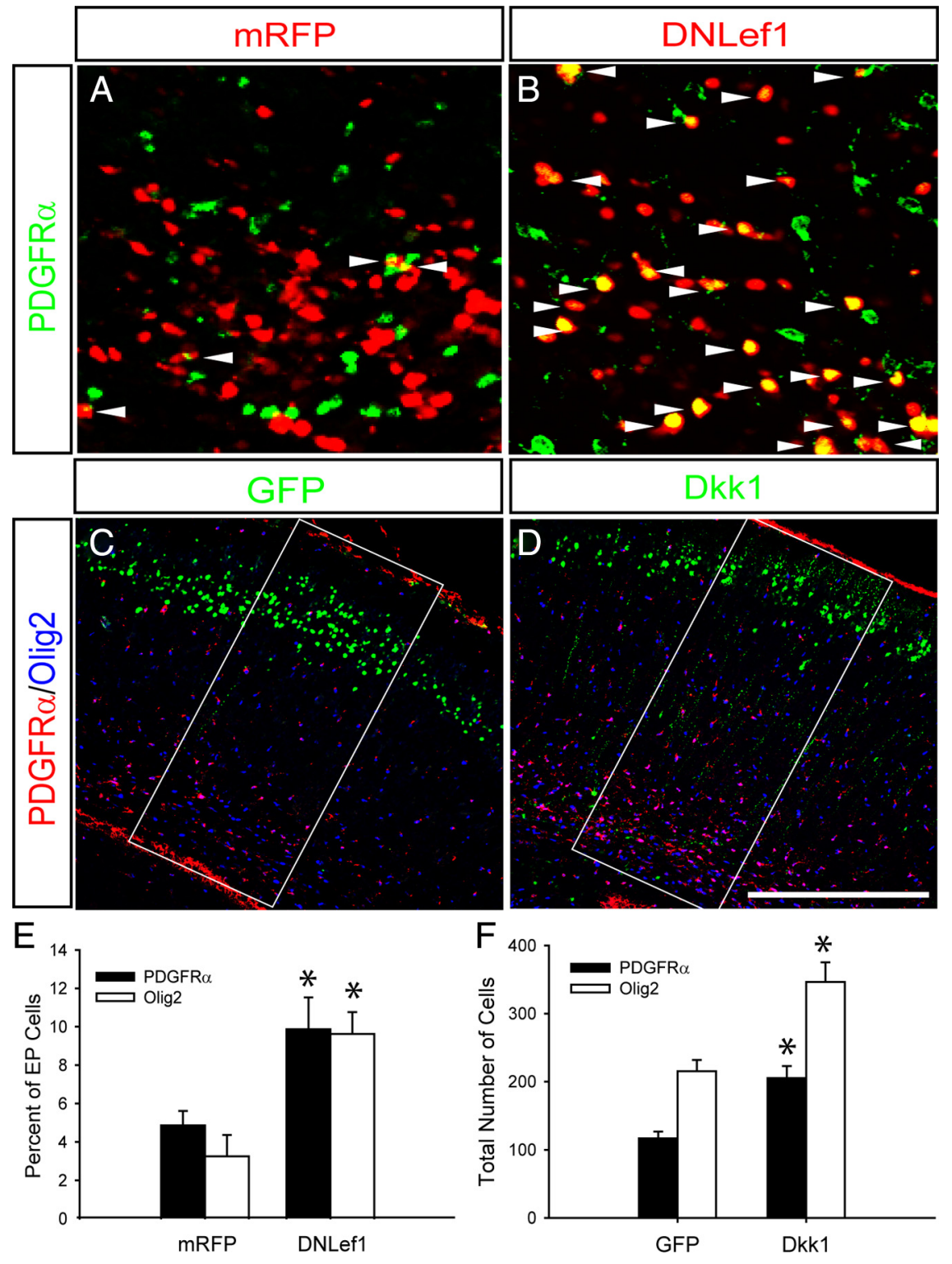

Figure 3. Decreasing Wnt tone in cortical progenitors results in a prescheduled increase in cortical OPC production. E15.5 EPs of mRFP control $(\boldsymbol{A})$ or DNLef1-mRFP $(\boldsymbol{B})$ into the lateral cortex were collected at PO. DNLef1 EP caused an increase in the percentage of EP PDGFR $\alpha$ - (DNLef1 $=9.87 \pm 1.66 \%$, RFP $=4.86 \pm 0.75 \%$ ) and Olig2- (not shown, DNLef1 $=9.62 \pm 1.13 \%$, RFP $=$ $3.24 \pm 1.11 \%$ ) positive cells. Arrowheads indicate EP cells that are PDGFR $\alpha$ positive. This is graphed in E. E15.5 EPs of GFP control $(\boldsymbol{C})$ or Dkk1-GFP (D) into the lateral cortex were collected at P2. A region of interest was selected in the center of the EP area (white box) and the total number of PDGFR $\alpha$ - or 0lig2-positive cells within the region was quantified. Scale bar, $500 \mu \mathrm{m}$. F, Dkk1 EP resulted in a significant increase in the number of PDGFR $\alpha$ - $(205.25 \pm 3.26)$ or 0lig2- $(346.50 \pm 28.83)$ positive cells in the EP region compared to control (116.75 \pm 9.92 PDGFR $\alpha$ and $215.50 \pm 16.250$ lig2). $N=3-4$ for all groups. ${ }^{*} p \leq 0.05$ for Student's

\section{Discussion}

Our study shows that Wnt signaling in progenitor cells influences the generation of OPCs in a cell-autonomous manner. Strong Wnt signaling inhibits OPC generation and low levels of Wnt signaling both accompany and drive OPC generation. This mechanism is used during development to influence the timing of OPC generation and is a significant factor influencing the efficiency of OPC production from neural precursor cells.

Decreased Wnt signaling accompanies the appearance of and drives production of OPCs

We have shown that Wnt signaling in the cortical progenitor domain is strong at embryonic ages when mostly neurons are 
produced and that there is a significant decrease in Wnt signaling when OPCs are generated. The addition of Wnt3a ligand to neural progenitor cultures causes a significant decrease in the percentage of OPCs produced from these progenitors, whereas addition of Dkk1 strongly increases the percentage of OPCs produced. This indicates that Wnt ligands and inhibitors are capable of acting directly on neural progenitors and that changes in the extracellular concentrations of Wnts and inhibitors can significantly influence the lineage decisions of progenitors. The lesser effect of Wnt3a compared to Dkk1 implies that significant Wnt ligand levels natively found in these culture systems are one inhibitor of OPC production.

Retroviral infection of DNLef1 into neural progenitors drove a significant increase in the percentage of OPCs produced from these infected progenitors without influencing the uninfected cells. This indicates that Wnt signaling acts autonomously to influence lineage decisions of individual progenitor cells, and that lower Wnt signaling levels favor the production of OPCs. Previous reports indicate that Wnt signaling drives proliferation in OPCs (Fancy et al., 2009; White et al., 2010) and blocks terminal differentiation during development and remyelination (Shimizu et al., 2005; Fancy et al., 2009; Ye et al., 2009). We did not see these effects in our neuronal progenitor cultures when we exposed them to Wnt ligand or inhibitors for short periods of time. However, when we exposed our cultures to Wnt3a ligand for $8 \mathrm{~d}$, we observed a $46 \%$ increase in the percentage of OPCs in the Wnt3a condition caused by a $257 \%$ increase in the proportion of proliferating OPCs (apoptosis of OPCs does not explain these changes) (supplemental Fig. 2, available at www.jneurosci.org as supplemental material).

To test the influence of Wnt signaling on the timing of OPC production from cortical progenitors, we electroporated a Dkk1-expressing construct into the progenitor zone of the lateral embryonic cortex at E15.5, before the time of cortical OPC production. At P2 the number of OPCs observed in the electroporated region was increased despite the potential for decreased proliferation of the earlier-born OPCs caused by decreased Wnt signaling (Fancy et al., 2009; White et al., 2010). This indicates that Wnts act on the cortical progenitors and that decreasing Wnt signaling in a physiologically relevant manner, without manipulating intracellular Wnt signaling components, influences the cell types produced from cortical progenitors.

We also determined that Wnt signaling acts on the cortical progenitors in a cell-autonomous manner by electroporating a DNLef1-expressing construct into the lateral cortical progenitor zone at E15.5 and examining OPC production $4 \mathrm{~d}$ later, at P0.5, when cortical production of OPCs normally begins (Kessaris et al., 2006). These studies show that shutting off the transcriptional output of the Wnt pathway in the primary progenitors of the embryonic cortex and their progeny significantly increases the percentage of electroporated cells becoming OPCs. This indicates that Wnt signaling acts cell autonomously on cortical progenitors to influence the production of OPCs. Whether Wnts also act to regulate migration of OPCs to the cortex is not something we explicitly examined in our studies; however, our electroporation experiments with DKK1 did not show any change in the overall distribution of OPCs. Further studies might be warranted to examine possible roles of Wnts in OPC migration.

Wnt signaling is not the only mechanism influencing the production of OPCs. We electroporated DNLef1 into the lateral cortical progenitor zone at E13.5 and analyzed the brains at E17.5, several days before the reported onset of cortical OPC production (Kessaris et al., 2006). We observed no significant difference in the percentage of electroporated cells positive for OPC markers (data not shown). This indicates that there are additional mechanisms inhibiting OPC production at earlier stages in development.

Our studies indicate that high Wnt signaling has contrasting effects on the oligodendrocyte lineage at different developmental stages - blocking specification of OPCs but driving the proliferation of already established OPCs [this latter finding is in agreement with previous publications (Fancy et al., 2009; White et al., 2010)]. In addition, Wnt signaling is known to inhibit OPC differentiation during development and remyelination (Shimizu et al., 2005; Fancy et al., 2009; Ye et al., 2009). Thus, Wnt signaling plays multiple roles at distinct points in the oligodendrocyte lineage. Possible mechanisms for the effects of Wnt signaling on OPC specification come from previous studies showing that Wnt signaling downregulates Olig2 gene expression in HCN cells (Ye et al., 2009) and induces the expression of the neurogenic gene NeuroD1 in adult hippocampal subgranular zone progenitors (Gao et al., 2009; Kuwabara et al., 2009). If a similar mechanism exists in the SVZ during development, it could strongly favor neuronal production.

\section{References}

Backman M, Machon O, Mygland L, van den Bout CJ, Zhong W, Taketo MM, Krauss S (2005) Effects of canonical Wnt signaling on dorsoventral specification of the mouse telencephalon. Dev Biol 279:155-168.

Chenn A (2008) Wnt/beta-catenin signaling in cerebral cortical development. Organogenesis 4:76-80.

Fancy SP, Baranzini SE, Zhao C, Yuk DI, Irvine KA, Kaing S, Sanai N, Franklin RJ, Rowitch DH (2009) Dysregulation of the Wnt pathway inhibits timely myelination and remyelination in the mammalian CNS. Genes Dev 23:1571-1585.

Franco CA, Liebner S, Gerhardt H (2009) Vascular morphogenesis: a Wnt for every vessel? Curr Opin Genet Dev 19:476-483.

Freese JL, Pino D, Pleasure SJ (2010) Wnt signaling in development and disease. Neurobiol Dis 38:148-153.

Gao Z, Ure K, Ables JL, Lagace DC, Nave KA, Goebbels S, Eisch AJ, Hsieh J (2009) Neurod1 is essential for the survival and maturation of adultborn neurons. Nat Neurosci 12:1090-1092.

Gulacsi AA, Anderson SA (2008) Beta-catenin-mediated Wnt signaling regulates neurogenesis in the ventral telencephalon. Nat Neurosci 11:1383-1391.

Kessaris N, Fogarty M, Iannarelli P, Grist M, Wegner M, Richardson WD (2006) Competing waves of oligodendrocytes in the forebrain and postnatal elimination of an embryonic lineage. Nat Neurosci 9:173-179.

Kim AS, Lowenstein DH, Pleasure SJ (2001) Wnt receptors and Wnt inhibitors are expressed in gradients in the developing telencephalon. Mech Dev 103:167-172.

Kuwabara T, Hsieh J, Muotri A, Yeo G, Warashina M, Lie DC, Moore L, Nakashima K, Asashima M, Gage FH (2009) Wnt-mediated activation of NeuroD1 and retro-elements during adult neurogenesis. Nat Neurosci 12:1097-1105.

Lee KJ, Jessell TM (1999) The specification of dorsal cell fates in the vertebrate central nervous system. Annu Rev Neurosci 22:261-294.

Lee SM, Tole S, Grove E, McMahon AP (2000) A local Wnt-3a signal is required for development of the mammalian hippocampus. Development 127:457-467.

Li G, Adesnik H, Li J, Long J, Nicoll RA, Rubenstein JL, Pleasure SJ (2008) Regional distribution of cortical interneurons and development of inhibitory tone are regulated by $\mathrm{Cxcl} 12 / \mathrm{Cxcr} 4$ signaling. J Neurosci 28:1085-1098.

Machon O, Backman M, Machonova O, Kozmik Z, Vacik T, Andersen L, Krauss S (2007) A dynamic gradient of Wnt signaling controls initiation of neurogenesis in the mammalian cortex and cellular specification in the hippocampus. Dev Biol 311:223-237. 
Maretto S, Cordenonsi M, Dupont S, Braghetta P, Broccoli V, Hassan AB, Volpin D, Bressan GM, Piccolo S (2003) Mapping Wnt/beta-catenin signaling during mouse development and in colorectal tumors. Proc Natl Acad Sci U S A 100:3299-3304.

Megason SG, McMahon AP (2002) A mitogen gradient of dorsal midline Wnts organizes growth in the CNS. Development 129:2087-2098.

Richardson WD, Kessaris N, Pringle N (2006) Oligodendrocyte wars. Nat Rev Neurosci 7:11-18.

Shimizu T, Kagawa T, Wada T, Muroyama Y, Takada S, Ikenaka K (2005) Wnt signaling controls the timing of oligodendrocyte development in the spinal cord. Dev Biol 282:397-410.

White BD, Nathe RJ, Maris DO, Nguyen NK, Goodson JM, Moon RT, Horner
PJ (2010) Beta-catenin signaling increases in proliferating NG2 + progenitors and astrocytes during post-traumatic gliogenesis in the adult brain. Stem Cells 28:297-307.

Ye F, Chen Y, Hoang T, Montgomery RL, Zhao XH, Bu H, Hu T, Taketo MM, van Es JH, Clevers H, Hsieh J, Bassel-Duby R, Olson EN, Lu QR (2009) HDAC1 and HDAC2 regulate oligodendrocyte differentiation by disrupting the beta-catenin-TCF interaction. Nat Neurosci 12:829-838.

Zhou CJ, Borello U, Rubenstein JL, Pleasure SJ (2006) Neuronal production and precursor proliferation defects in the neocortex of mice with loss of function in the canonical Wnt signaling pathway. Neuroscience 142: 1119-1131. 